\title{
Glycyrrhetinic Acid Induces Anoikis-Like Death and Cytoskeletal Disruption in the Central Nervous System Tumorigenic Cells
}

\author{
Hideaki Yamaguchi, ${ }^{* a,{ }^{\#}}$ Yumi KidachI, ${ }^{b, \#}$ Katsuyoshi KamiIE, ${ }^{b}$ Toshiro Noshita, ${ }^{c}$ \\ Hironori UMETSU, ${ }^{d}$ and Kazuo RYOYAMA ${ }^{b, e}$ \\ ${ }^{a}$ Department of Pharmacy, Faculty of Pharmacy, Meijo University; 150 Yagotoyama, Tenpaku, Nagoya 468-8503, Japan: \\ ${ }^{b}$ Department of Clinical Pharmacy, Faculty of Pharmaceutical Sciences, Aomori University; ${ }^{e}$ Graduate School of \\ Environmental Sciences, Aomori University; 2-3-1 Kobata, Aomori 030-0943, Japan: ${ }^{\circ}$ Department of Life Sciences, \\ Faculty of Life and Environmental Sciences, Prefectural University of Hiroshima; 562 Nanatsuka, Shobara 727-0023, \\ Japan: and ${ }^{d}$ Laboratory of Food Chemistry, Department of Life Sciences, Junior College, Gifu Shotoku Gakuen \\ University; 1-38 Nakauzura, Gifu 500-8288, Japan.
}

Received September 3, 2009; accepted November 4, 2009; published online November 10, 2009

We analyzed the effects of glycyrrhetinic acid (GA), a licorice compound, on the induction of anoikis-like death and cytoskeletal disruption in the central nervous system (CNS) tumorigenic cells. GA was cytotoxic in time- and dose-dependent manners, and the tumorigenic cells shed floating cells upon the GA treatment and even some of the adherent cells were easily detached from the fibronectin-coated culture dish by gentle shaking and aspiration. Reculture of the detached cells revealed that the longer the duration of GA exposure, the less the number of the proliferatable cells. These results indicate that GA perturbs cell adhesion and induces anoikis-like cell death. Further, GA also induced morphologic changes and disturbed cytoskeletal proteins.

Key words glycyrrhetinic acid; licorice; tumor; human c-Ha-ras mouse c-myc cotransfected highly metastatic serum-free mouse embryo-1

Licorice extracts and the principal licorice component glycyrrhizin (GL) are used worldwide in foods, tobacco and medicines. The sweet taste of licorice roots arises from GL, which is reputed to be at least 50 times sweeter than refined sugar. Owing to this sweetness, GL is extensively used as a natural sweetener and flavoring additive. ${ }^{1)} \mathrm{GL}$ is a saponin compound comprising a triterpenoid aglycone, glycyrrhetinic acid (GA), conjugated to a disaccharide of glucuronic acid. GA is used as an antitoxic and immunological regulatory agent for the prevention or treatment of viral infection, inflammation and anaphylaxis. ${ }^{2,3)}$ However, despite its wide use in the market and great effects on some physiological aspects, to the best of our knowledge, no studies have investigated the antitumor effects of GA on tumor cells in the central nervous system (CNS).

Serum-free mouse embryo (SFME) cells were originally derived from a 16-d-old whole Balb/c mouse embryo and are maintained in a serum-free culture medium. ${ }^{4)}$ These cells do not undergo growth crisis, maintain their diploid karyotype for extended passages and are non-tumorigenic in vivo. Consequently, they are non-transformed, behave as primary cultures, have a finite lifespan and display the characteristics of CNS progenitor cells. ${ }^{5,6)}$ SFME cells were cotransfected with the human c-Ha-ras and mouse c-myc genes, and the resulting cells were designated ras/myc SFME cells. ${ }^{7)}$ While SFME cells are non-tumorigenic in vivo, ${ }^{5,6)}$ ras/myc SFME cells are tumorigenic and do not require any growth factors, such as epidermal growth factor. ${ }^{7)}$ Another line of SFME-derived tumorigenic cells are highly metastatic ras/myc SFME$1(r / m$ HM-SFME-1) cells, which were established by selecting ras/myc SFME cells that only metastasize to the lungs of $\mathrm{Balb} / \mathrm{c}$ mice. ${ }^{8)}$

In the present study, $r / m$ HM-SFME-1 cells were treated with GA and its efficacy as an antitumor agent through anoikis-like cell death and cytoskeletal disruption was inves- tigated.

\section{MATERIALS AND METHODS}

Materials GA was purchased from Wako Pure Chemical Industries Ltd. (Osaka, Japan). Mouse monoclonal anti- $\beta$ actin, mouse monoclonal anti-glyceraldehyde-3-phosphatedehydrogenase (GAPDH), mouse monoclonal anti- $\beta$ III-tubulin, AP-conjugated anti-mouse immunoglobulin $\left(\operatorname{IgG}_{1}\right)$ and HiLyte Fluor ${ }^{\mathrm{TM}}$ 488-conjugated goat anti-mouse $\mathrm{IgG}$ antibodies were obtained from Sigma (St. Louis, MO, U.S.A.), Santa Cruz Biotechnology (Santa Cruz, CA, U.S.A.), R\&D Systems Inc. (Minneapolis, MN, U.S.A.), Chemicon International (Temecula, CA, U.S.A.) and AnaSpec Inc. (San Jose, CA, U.S.A.), respectively.

Cell Culture SFME and $r / m$ HM-SFME-1 cells were cultured in a humidified $5 \% \mathrm{CO}_{2}-95 \%$ air atmosphere at $37^{\circ} \mathrm{C}$ in $60 \mathrm{~mm}$ diameter dishes, pre-coated with $10 \mu \mathrm{g} / \mathrm{ml} \mathrm{fi-}$ bronectin. The basal nutrient culture medium was a $1: 1 \mathrm{mix}$ ture of Dulbecco's modified Eagle's medium and Ham's F12 ${ }^{9,10)}$ containing $15 \mathrm{~mm} N$-(2-hydroxyethyl)piperazine- $N^{\prime}$ 2-ethanesulfonic acid (HEPES), pH 7.4, $1.2 \mathrm{mg} / \mathrm{ml}$ sodium bicarbonate, $10 \mathrm{~nm}$ sodium selenite and $10 \mu \mathrm{g} / \mathrm{ml}$ gentamicin, supplemented with insulin $(10 \mu \mathrm{g} / \mathrm{ml})$, transferrin $(25 \mu \mathrm{g} / \mathrm{ml})$ and epidermal growth factor $(50 \mathrm{ng} / \mathrm{ml})$. Cell passages were accomplished by rapid trypsinization with $0.2 \%$ crude trypsin and $1 \mathrm{~mm}$ ethylenediaminetetraacetate in phosphatebuffered saline (PBS) without calcium or magnesium, followed by dilution in the culture medium at room temperature. The medium containing the collected cells was centrifuged at $250 \boldsymbol{g}$ at $4{ }^{\circ} \mathrm{C}$ for $7 \mathrm{~min}$ and the supernatant was removed. The cells were suspended in the culture medium without the supplements, plated at $1 \times 10^{5}$ cells/dish and cultured again in the medium with the supplements. Following the preincubation, the cells were treated with various concen- 
trations of GA at half confluency. After culture for another $0.5,2,8$ and $24 \mathrm{~h}$, the cells were analyzed by the $3-(4,5-\mathrm{di}-$ methylthiazol-2-yl)-2,5-diphenyltetrazolium bromide (MTT) assay $^{11)}$ and microscopic observations. Upon GA treatment, some cells were detached from the culture dishes and then recultured for viability assays. Cell viability was also analyzed by the MTT assay ${ }^{11)}$ and microscopic observations.

Western Blotting Analysis Proteins were extracted with PBS containing $1 \mathrm{~mm}$ phenylmethylsulfonyl fluoride (PMSF), $1 \mathrm{~mm}$ ethylenediaminetetraacetic acid (EDTA), $2 \mathrm{~mm}$ 2-mercaptoethanol and $1 \%$ Triton $\mathrm{X}-100$ at $4{ }^{\circ} \mathrm{C}$ for $3.5 \mathrm{~h}$. For western blotting analysis, aliquots of proteins were separated by sodium dodecyl sulfate-polyacrylamide gel electrophoresis (SDS-PAGE), blotted onto a nitrocellulose membrane and probed with a primary antibody followed by a secondary antibody. The primary antibodies used were: mouse monoclonal anti- $\beta$-actin (Sigma, St. Louis, MO, U.S.A.); mouse monoclonal anti-GAPDH (Santa Cruz Biotechnology, Santa Cruz, CA, U.S.A.); and mouse monoclonal anti- $\beta$ III-tubulin (R\&D Systems Inc., Minneapolis, MN, U.S.A.). The secondary antibody used was AP-conjugated anti-mouse $\mathrm{IgG}_{1}$ (Chemicon International, Temecula, CA, U.S.A.). Visualization of the antigen-antibody complexes was performed with $33 \mu \mathrm{l}$ of 5-bromo-4-chloro-3-indolyl phosphate, $66 \mu \mathrm{l}$ of nitroblue tetrazolium and $40 \mu \mathrm{l}$ of $1 \mathrm{M} \mathrm{MgCl}_{2}$ in $10 \mathrm{ml}$ of $0.1 \mathrm{M}$ Tris-HCl buffer ( $\mathrm{pH}$ 9.5). Images of the positive bands were obtained by scanning and the densities were determined using an LAS-3000 image analyzer (Fuji Film, Tokyo, Japan).

Immunofluorescence Staining and Confocal LaserScanning Microscopic Observation Cells were fixed with $3.7 \%$ formaldehyde in PBS and permeabilized in PBS containing $0.1 \%$ Triton X-100. For F-actin labeling, cells were incubated with rhodamine-phalloidin. As previously reported, ${ }^{12)}$ the anti- $\beta$ III-tubulin antibody in the present study showed appropriate specificities (data not shown). For $\beta$ IIItubulin labeling, cells were blocked in Tris-buffered saline containing $0.05 \%$ Tween-20 and $3 \%$ non-fat dried milk. Next, the cells were incubated with a mouse monoclonal anti- $\beta$ III-tubulin antibody, washed with PBS and incubated with HiLyte Fluor ${ }^{\mathrm{TM}}$ 488-conjugated goat anti-mouse IgG. After washing and mounting with ProLong Gold anti-fade reagent (Molecular Probes, Eugene, OR, U.S.A.), the cells were observed by confocal microscopy using an LSM510 META confocal laser scanning microscope equipped with Ar and He-Ne lasers (Carl Zeiss Japan, Tokyo, Japan) or a BIOREVO BZ-9000 fluorescence microscope system (Keyence, Osaka, Japan). Images were captured using $40 \times$, $63 \times$ and $100 \times$ oil immersion objective lenses, analyzed and processed with the software Image Browser (Carl Zeiss Japan).

Statistical Analysis Experiments were performed in triplicate and repeated at least six times. The values quoted are given as means \pm S.D. Ordinary or repeated-measures analysis of variance (ANOVA) followed by Dunnett's multiple comparison test was used to evaluate the statistical significance of differences between multiple groups. Differences were considered significant at the level of $p<0.05$.

\section{RESULTS AND DISCUSSION}

Cytotoxic Effects of GA on $\boldsymbol{r} / \boldsymbol{m}$ HM-SFME-1 Cells In the present study, $r / m$ HM-SFME-1 cells were treated with GA and its efficacy as an antitumor agent was investigated. As shown in Fig. 1A, the tumor cells proliferated time-dependently without GA and with the GA treatment at $2.5 \mu \mathrm{M}$. In contrast, the cell proliferation exhibited a time-dependent inhibition by the GA treatment at 10 and $20 \mu \mathrm{M}$. The cell growth of the tumor cells over $0.5 \mathrm{~h}$ of treatment with various concentrations of GA revealed that about 30 and $50 \%$ of the proliferative capability of the tumor cells were inhibited by GA at 10 and $20 \mu \mathrm{M}$, respectively. GA was also cytotoxic in time- and dose-dependent manners and it reduced the cell growth by less than $20 \%$ at 10 and $20 \mu \mathrm{M}$ by $24 \mathrm{~h}$. Then, normal SFME and tumorigenic $r / m$ HM-SFME-1 cells were treated with $10 \mu \mathrm{M}$ GA for $0.5,2,8$ and $24 \mathrm{~h}$ to investigate its effects on cell growth (Fig. 1B). More than $75 \%$ of the normal cells survived, and in contrast only about $20 \%$ of the tumor cells survived over the 24-h GA treatment. These re-
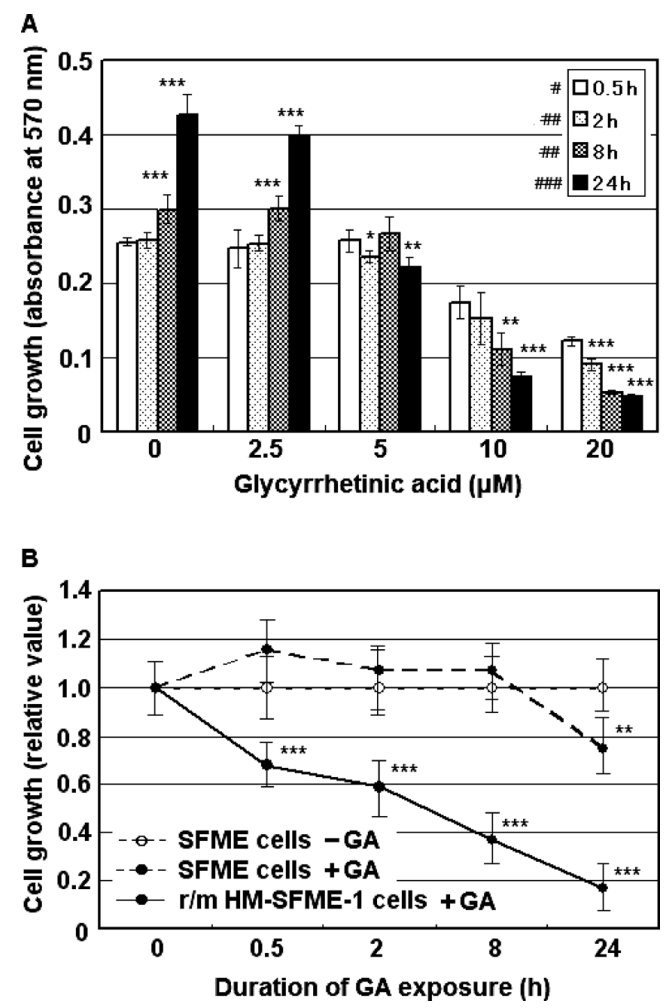

Fig. 1. GA Is Cytotoxic in Time- and Dose-Dependent Manners and Reduces Cell Growth of Tumorigenic $r / m$ HM-SFME-1 Cells

(A) Cell growth of the tumor cells over $0.5,2,8$ and $24 \mathrm{~h}$ of treatment with various concentrations of GA was analyzed. The cell growth increases time-dependently at 0 and $2.5 \mu \mathrm{M}$ of GA treatment. In contrast, it decreases time-dependently at 10 and $20 \mu \mathrm{M}$ GA treatment. Significant cell growth inhibition is found at and more than $10 \mu \mathrm{M}$ GA treatment with $0.5 \mathrm{~h}$ exposure (open bar), while it is found at and more than $2.5 \mu \mathrm{M} \mathrm{GA}$ treatment with $24 \mathrm{~h}$ exposure (closed bar). Cell growth was measured by the MTT method. Cytotoxicity was calculated by the cell numbers of each treatment against those of the control cells, measured by the MTT method. Each point is the mean of at least 6 experiments. *,** and $* * * p<0.05, p<0.01$ and $p<0.001$, respectively by Dunnett's test compared with the different GA-exposure time. \#, \# and \#\# Significance of difference at $p<0.05$ (Dunnett's test) was found at and more than 10, 5 and $2.5 \mu \mathrm{M} \mathrm{GA}$ treatment, respectively. (B) Normal SFME and tumorigenic $r / m$ HM-SFME-1 cells were treated with $10 \mu \mathrm{M}$ GA for $0.5,2,8$ and $24 \mathrm{~h}$ to investigate its effects on cell growth. Cell growth was measured by the MTT method. More than $75 \%$ of the normal cells survive, and in contrast only about $20 \%$ of the tumor cells survive over the $24-\mathrm{h}$ GA treatment. Each point is the mean \pm S.D. of at least 6 experiments. ** and $* * * p<0.01$ and $p<0.001$, respectively by Dunnett's test. 
sults indicate that GA is capable of effectively killing tumor cells in the CNS.

Viability of the GA-Treated $r / m$ HM-SFME-1 Cells upon Reculture Interestingly, the tumor cells shed floating cells upon the GA treatment and even some of the adherent cells were easily detached from the fibronectin-coated culture dish by gentle shaking and aspiration. Therefore, we treated $\mathrm{r} / \mathrm{m}$ HM-SFME- 1 cells with $10 \mu \mathrm{M}$ GA, collected the detached cells and recultured them without GA to examine whether or not this adhesion loss caused by GA is fatal and irreversible. Although an immediate non-GA reculture of the detached cells could save them from death, detached but proliferatable cells were about $30 \%$ of the control cells with $0.5 \mathrm{~h}$-pre-exposure of $10 \mu \mathrm{M}$ GA, and they lost their viability with 8 h-pre-exposure (Fig. 2). These results indicate that the longer the duration of GA exposure upon the tumor cells, the less the chances of their survival, and that eventually the adhesion loss and cell detachment caused by GA leads to complete cell death.

Morphologic Effects of GA on $r / m$ HM-SFME-1 Cells Next, $r / m$ HM-SFME- 1 cells were exposed to $10 \mu \mathrm{M}$ GA for 8 and $24 \mathrm{~h}$ and the cells were observed microscopically to analyze the morphologic effects of GA on the tumor cells. As shown in Fig. 3A in the upper panels, the tumor cells proliferated vigorously without GA treatment. In contrast, the GAtreated tumor cells at $8 \mathrm{~h}$ stopped cell growth and exhibited loss of adherence and round shapes with cell shrinkage (Fig. $3 \mathrm{~A}$, lower middle panel), and obvious cell death was found at $24 \mathrm{~h}$ (Fig. 3A, lower right panel). The morphologic changes typical of apoptosis, such as cell shrinkage and blebbing, were also detected (data not shown). These morphologic changes caused by GA seemed concomitant with the adhesion loss, which suggests that the GA-treated cell death is not only caused by the consequence of apoptotic signals but also induced by the detachment from extracellular matrix (ECM) substrates. The induction of apoptosis through the detachment from ECM has been know as "anoikis."13)

Effects of GA on Cytoskeletal Proteins in $r / m$ HMSFME-1 Cells The results of the morphologic and anoikislike effects by GA in the present study prompted us to ana-

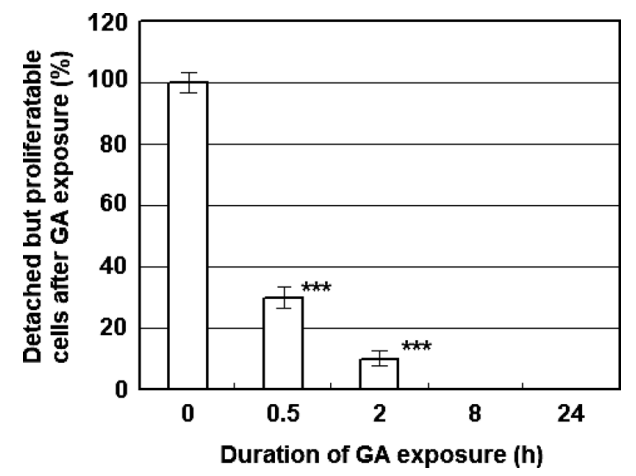

Fig. 2. Reculture of the Detached Cells Reveals That the Longer the Duration of GA Exposure, the Less the Number of the Proliferatable Cells

$r / m$ HM-SFME-1 cells were treated with $10 \mu \mathrm{M} \mathrm{GA}$, and the detached cells were collected and recultured without GA to examine whether or not the adhesion loss caused by GA is fatal and irreversible. Detached but proliferatable cells are about $30 \%$ of the control cells with $0.5 \mathrm{~h}$-pre-exposure of $10 \mu \mathrm{M} \mathrm{GA}$, and they lost their viability with $8 \mathrm{~h}$ pre-exposure. Cell proliferation was measured by the MTT method. Cytotoxicity was calculated by the cell numbers of each treatment against those of the control cells, measured by the MTT method. Each point is the mean of at least 6 experiments. $* * * p<0.001$ by Dunnett's test compared with the duration of GA-exposure. lyze the actin and microtubule cytoskeletons, since together they provide the cell shape and maintain the cellular structure $^{14)}$ and disruption of structural elements might trigger anoikis. ${ }^{15)}$ After GA treatment at $10 \mu \mathrm{M}$ for 8 and $24 \mathrm{~h}, \mathrm{~F}$ actin and $\beta$ III-tubulin were analyzed by immunofluorescence staining and confocal laser-scanning microscopic observation. As shown in Fig. 3B in the middle left panel, the tumor cells showed broad lamellipodia and displayed broad F-actin extensions. The GA-treated cells exhibited disappearance of the F-actin extensions at $8 \mathrm{~h}$ (Fig. 3B, middle middle panel), and instead the staining was rather granulated and condensed at $24 \mathrm{~h}$ (Fig. 3B, middle right panel). The loss of F-actin ex-
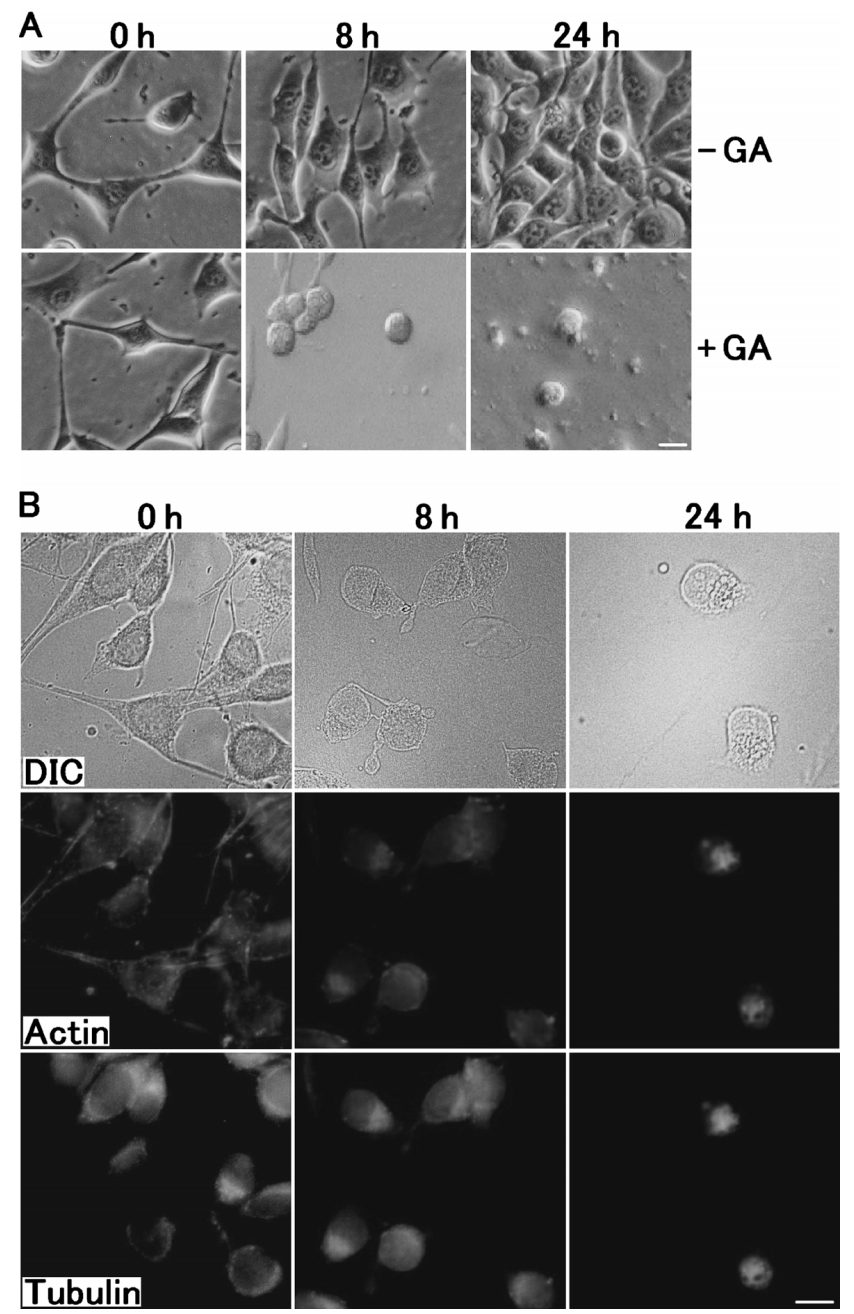

Fig. 3. GA Induces Morphologic Changes and Disturbances in Cytoskeletal Proteins and Causes Tumor Cell Death

(A) Microscopic observations of $r / m$ HM-SFME-1 cells. The tumor cells were exposed to $10 \mu \mathrm{M}$ GA for 8 and $24 \mathrm{~h}$. The cells exposed to GA for $8 \mathrm{~h}$ show loss of adherence and exhibit round shapes (lower middle panel). By $24 \mathrm{~h}$, the GA-exposed cells lose their viability (lower right panel). Bar, $20 \mu \mathrm{m}$. (B) Fluorescence microscopic observations of $r / m$ HM-SFME- 1 cells. The tumor cells were exposed to $10 \mu \mathrm{M}$ GA for 8 and $24 \mathrm{~h}$. In the middle middle panel, the 8-h GA-treated tumor cells exhibit disappearance of the F-actin extensions, and instead the staining is rather granulated and condensed. Loss of the F-actin extensions at the periphery of the cell membrane is particularly obvious and the tumor cells are no longer capable of maintaining the characteristic broad lamellipodia. The 24-h GA-treated tumor cells exhibit further progression of cell shrinkage and loss of F-actin extensions, and the characteristic broad lamellipodia have completely disappeared (middle right panel). In the lower left panel, $\beta$ III-tubulin staining in the control cells is homogeneous and mainly located in the cytoplasm. In contrast, in the lower right panel, the 24-h GA-treated cells show non-homogeneous $\beta$ IIItubulin staining and voids or puncture-like disorganizations of $\beta$ III-tubulin are observed in the cytoplasm. Upper panels, differential interference contrast (DIC) image; Middle panels, detected F-actin; Lower panels, detected $\beta$ III-tubulin. Bar, $20 \mu \mathrm{m}$. 

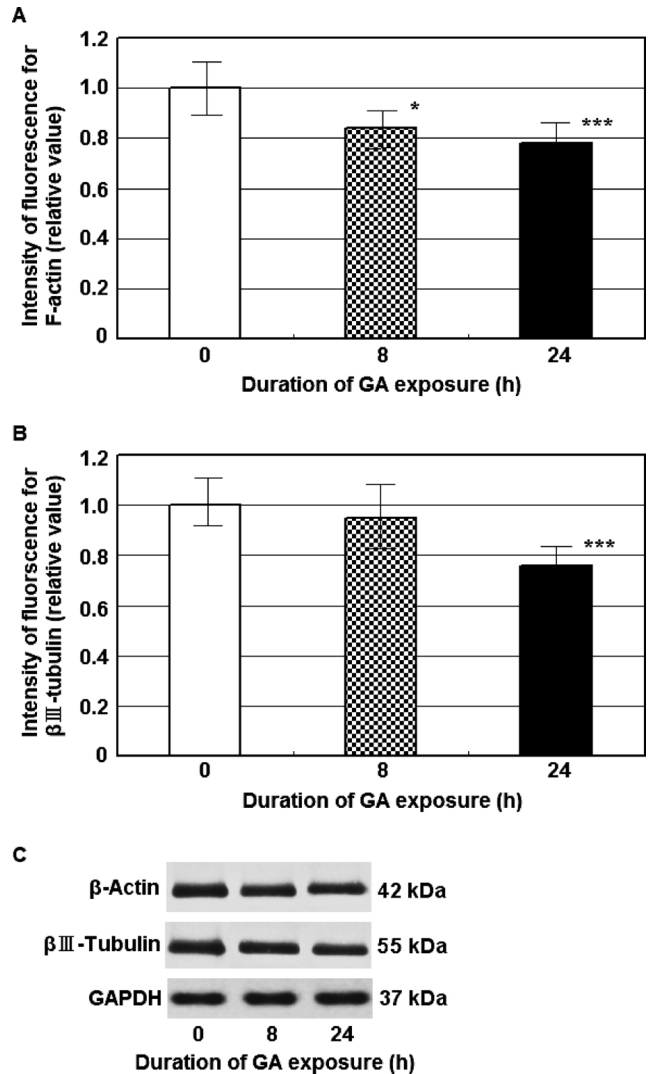

Fig. 4. Relative Intensity of Immunofluorescence and Western Blotting Analysis for Actin and Tubulin in Tumor Cells

$r / m$ HM-SFME-1 cells were exposed to $10 \mu \mathrm{M}$ GA for 8 and $24 \mathrm{~h}$. Both F-actin (A) and $\beta$ III-tubulin (B) expressions are significantly downregulated in the 24-h GA-treated tumor cells. Each point is the mean \pm S.D. of at least 6 experiments. $*$ and $* * * p<0.05$ and $p<0.001$ by Dunnett's test compared with the duration of GA-exposure. (C) Downregulation of $\beta$-actin and $\beta$ III-tubulin is also confirmed by Western blotting analyses. GAPDH, a loading control.

tensions at the periphery of the cell membrane was particularly obvious and the cells were no longer capable of maintaining the characteristic broad lamellipodia. These results indicate that GA disrupts the actin cytoskeleton in the tumor cells. $\beta$ III-Tubulin staining in the control cells was homogeneous and mainly located in the cytoplasm (Fig. 3B, lower left panel). In contrast, the 24-h GA treatment revealed nonhomogeneous $\beta$ III-tubulin staining and the staining was also rather condensed. Voids or puncture-like disorganizations of $\beta$ III-tubulin were also observed in the cytoplasm (Fig. 3B, lower right panel). Relative intensity of immunofluorescence for $\mathrm{F}$-acin and $\beta \mathrm{III}$-tubulin was also analyzed. Expression of F-acin was significantly decreased at 8 and $24 \mathrm{~h}$ (Fig. 4A) and the significant decrease in $\beta$ III-tubulin was found at $24 \mathrm{~h}$ (Fig. 4B) in the GA-treated tumor cells. Those were also confirmed by western blotting analyses (Fig. 4C). These results indicate that GA causes disturbances in these cytoskeletal proteins, which could lead to loss of functionality for cell structure and result in the anoikis-like cell death. It has been reported that interference with actin or microtubule functions associated with the integrity of the cytoskeleton, even with- out affecting the amount of the cytoskeletal proteins, could be utilized as a strategy for developing novel antitumor treatments ${ }^{14)}$ which suggests that qualitative changes in the cytoskeleton are of very importance. In fact, our results are in agreement with the previous report which suggested that disruption of cytoskeletal proteins was associated with the induction of anoikis in cancer cells. ${ }^{16)}$

In conclusion, we provide evidence that GA is capable of effectively killing tumor cells in the CNS, that adhesion loss caused by GA is fatal and irreversible, and that GA induces cytoskeletal disruption and anoikis-like death. Further, the GA content in licorice has been reported to be 5.8 to $11.4 \%,{ }^{17)}$ and plasma GA levels reached $10 \mu \mathrm{M}$ in humans ingesting licorice, ${ }^{18)}$ which is exactly the same concentration that affected the tumor cells effectively in the present study. Although validation studies supporting the utilization in clinical practice are warranted, a licorice compound GA, that could disturb cytoskeletons and adhesion, may potentiate anticancer effects on the anoikis-prone cells.

Acknowledgments We thank Dr. S. Shirahata (Kyushu University, Fukuoka, Japan) for the gift of the SFME cells. This study was partially supported by a grant-in-aid from the Promotion and Mutual Aid Corporation for Private Schools of Japan.

\section{REFERENCES}

1) Isbrucker R. A., Burdock G. A., Regul. Toxicol. Pharmacol., 46, 167-192 (2006).

2) Shibata S., Yakugaku Zasshi, 120, 849-862 (2000).

3) Armanini D., Fiore C., Mattarello M. J., Bielenberg J., Palermo M., Exp. Clin. Endocrinol. Diabetes, 110, 257-261 (2002).

4) Loo D. T., Fuquay J. I., Rawson C. L., Barnes D. W., Science, 236, 200-202 (1987).

5) Loo D., Rawson C., Helmrich A., Barnes D., J. Cell. Physiol., 139, 484-491 (1989).

6) Rawson C., Cosola-Smith C., Barnes D., Exp. Cell Res., 186, 177181 (1990).

7) Rawson C., Shirahata S., Collodi P., Natsuno T., Barnes D., Oncogene, 6, 487-489 (1991).

8) Nomura T., Matano S., Okada G., Tokuyama H., Hori I., Nakamura S., Kameyama T., Ryoyama K., In Vitro Cell. Dev. Biol., 29A, 614-616 (1993).

9) Ham R. G., McKeehan W. L., Methods Enzymol., 58, 44-93 (1979).

10) Mather J. P., Sato G. H., Exp. Cell Res., 124, 215-221 (1979).

11) Carmichael J., DeGraff W. G., Gazdar A. F., Minna J. D., Mitchell J. B., Cancer Res., 47, 943-946 (1987).

12) Yamaguchi H., Kidachi Y., Umetsu H., Ryoyama K., Biol. Pharm. Bull., 31, 1008-1012 (2008).

13) Frisch S. M., Francis H., J. Cell Biol., 124, 619-626 (1994).

14) Bijman M. N., van Berkel M. P., van Nieuw Amerongen G. P., Boven E., Biochem. Pharmacol., 76, 707-716 (2008).

15) Flusberg D. A., Numaguchi Y., Ingber D. E., Mol. Biol. Cell, 12, 3087-3094 (2001).

16) Deschesnes R. G., Patenaude A., Rousseau J. L. C., Fortin J. S., Ricard C., Cote M. F., Huot J., C.-Gaudreault R., Petitclerc E., J. Pharmacol. Exp. Ther, 320, 853-864 (2007).

17) Gu H., Gong L., Yu J., J. Forest. Res., 13, 141-143 (2002).

18) de Groot G., Koops R., Hogendoorn E. A., Goewie C. E., Savelkoul T. J., van Vloten P., J. Chromatogr., 456, 71-81 (1988). 